\title{
Prototype Nerve-Specific Near-Infrared Fluorophores
}

\author{
Min Ho Park ${ }^{1,2^{*}}$, Hoon Hyun ${ }^{*}$, Yoshitomo Ashitate ${ }^{1,3}$, Hideyuki Wada1,3, GwangLi Park ${ }^{1}$, Jeong Heon Lee ${ }^{1}$, \\ Costyl Njiojob ${ }^{4}$, Maged Henary ${ }^{4}$, John V. Frangioni1 ${ }^{1,5,6}$, Hak Soo Choi ${ }^{1,7}{ }^{凶}$
}

1. Division of Hematology/Oncology, Department of Medicine, Beth Israel Deaconess Medical Center and Harvard Medical School, Boston, MA 02215.

2. Department of Surgery, Chonnam National University Medical School, Gwangju 501-746, South Korea.

3. Department of Gastroenterological Surgery II, Hokkaido University Graduate School of Medicine, Sapporo 060-8638, Japan.

4. Department of Chemistry, Georgia State University, Atlanta, GA 30303.

5. Department of Radiology, Beth Israel Deaconess Medical Center and Harvard Medical School, Boston, MA 02215.

6. Curadel, LLC, 377 Plantation Street, Worcester, MA 01605.

7. Department of Cogno-Mechatronics Engineering, Pusan National University, Busan 609-735, South Korea.

*These authors contributed equally to this work.

$\triangle$ Corresponding author: Hak Soo Choi, Ph.D. BIDMC, Room SL-436A, 330 Brookline Avenue, Boston, MA 02215. Office: 617-667-6024; Fax: 617-975-5016. Email: hchoi@bidmc.harvard.edu.

() Ivyspring International Publisher. This is an open-access article distributed under the terms of the Creative Commons License (http://creativecommons.org/ licenses/by-nc-nd/3.0/). Reproduction is permitted for personal, noncommercial use, provided that the article is in whole, unmodified, and properly cited.

Received: 2014.01.27; Accepted: 2014.05.02; Published: 2014.06.07

\begin{abstract}
Nerve preservation is an important issue during most surgery because accidental transection or injury results in significant morbidity, including numbness, pain, weakness, or paralysis. Currently, nerves are still identified only by gross appearance and anatomical location during surgery, without intraoperative image guidance. Near-infrared (NIR) fluorescent light, in the wavelength range of 650-900 nm, has the potential to provide high-resolution, high-sensitivity, and real-time avoidance of nerve damage, but only if nerve-specific NIR fluorophores can be developed. In this study, we evaluated a series of Oxazine derivatives to highlight various peripheral nerve structures in small and large animals. Among the targeted fluorophores, Oxazine 4 has peak emission near into the NIR, which provided nerve-targeted signal in the brachial plexus and sciatic nerve for up to $12 \mathrm{~h}$ after a single intravenous injection. In addition, recurrent laryngeal nerves were successfully identified and highlighted in real time in swine, which could be preserved during the course of thyroid resection. Although optical properties of these agents are not yet optimal, chemical structure analysis provides a basis for improving these prototype nerve-specific NIR fluorophores even further.
\end{abstract}

Key words: Real-time intraoperative identification, nerve targeting, recurrent laryngeal nerve, contrast agents, targeted agents, near-infrared imaging.

\section{Introduction}

Nerve damage during surgery results in significant morbidity and mortality [1-4]. Despite advancements in surgical technique and equipment, nerves are currently identified via gross appearance and anatomical location, without intraoperative image guidance. Thin or buried nerves are at high risk for damage. Anatomical variants such as bifurcation, trifurcation, medial or anterior displacement, and non-recurrence are also risk factors for injury $[5,6]$. For example, recurrent laryngeal nerve (RLN) injury in the neck can cause symptoms ranging from hoarseness and aspiration in unilateral injury, to life threatening airway obstruction in the case of bilateral injury [2,7]. To minimize such high morbidity and mortality from nerve injury, intraoperative visual identification during complex surgery is of para- 
mount importance.

However, only 3 main classes of nerve-specific molecules have been reported to date, including stilbene derivatives, distyrylbenzene (DSB) fluorophores, and styryl pyridinium (FM) fluorophores [8-11]. Among these agents, DSBs such as BMB, GE3082, and GE3111 have been investigated for image-guided nerve surgery [12-14]. However, none of these compounds were optimal because of high adsorption and scattering in tissues from the ultraviolet and visible wavelengths required. In addition, due to their intrinsically high lipophilicity and charge, they exhibit high background binding to adipose tissues, thus lowering the signal-to-background ratio (SBR) considerably. Relatively high endogenous tissue autofluorescence in the visible wavelength range also limits the use of these fluorophores in vivo [15].

To overcome these limitations, nerve-specific contrast agents in the near-infrared (NIR) range are desperately needed. Recently, Whitney et al. synthesized peptides that localize to nerve-associated connective tissues [16]. They highlighted almost all nerves in the body including motor and sensory nerves, but only after labeling with exogenous fluorophores [16,17]. Gustafson et al. developed a NIR fluorophore, which is hydrophilic indocyanine green (ICG) analogue, for peripheral nerve targeting [18]. Gibbs et al. investigated the structure-activity relationship of over 200 nerve-specific contrast agents and also screened these molecules for NIR optical properties. Although no NIR agents were obtained, several putative fingerprints for nerve uptake were identified [13]. Based on these results, we investigated Oxazine-based chemical structures for simultaneous nerve uptake and NIR fluorescence. Our goal is to develop nerve-specific agents in the NIR range [19], which permits color video and NIR to be acquired simultaneously, thus providing real time image-guidance to surgeons about nerve location.

\section{Materials and Methods}

Optical Property Measurements. Oxazine derivatives were obtained from Fisher Scientific Inc. (Pittsburgh, PA) or Sigma-Aldrich (Saint Louis, MO), and BMB was synthesized as described previously [20]. Optical measurements were performed at $37^{\circ} \mathrm{C}$ in phosphate-buffered saline (PBS), pH 7.4 or $100 \%$ fetal bovine serum (FBS) buffered with $50 \mathrm{mM}$ HEPES, pH 7.4. Absorbance and fluorescence emission spectra were measured using fiber optic HR2000 absorbance (200-1100 nm) and USB2000FL fluorescence (350-1000 nm) spectrometers (Ocean Optics, Dunedin, FL). A $532 \mathrm{~nm}$ green laser pointer (Opcom Inc., Xiamen, China) set to $5 \mathrm{~mW}$ and coupled through a $300 \mu \mathrm{m}$ core diameter, NA 0.22 fiber (Fiberguide
Industries, Stirling, NJ) was used to excite fluorophores and avoid spectral overlapping between the laser excitation and fluorescence emission. In silico calculation of the partition coefficient $(\log \mathrm{D}$ at $\mathrm{pH} 7.4)$ was calculated using Marvin and JChem calculator plugins (ChemAxon, Budapest, Hungary). Chemical similarities were calculated by Instant JChem 6.04 using "Chemical Hashed Fingerprint" with the Normalized Euclidean dissimilarity screening configuration (ChemAxon).

NIR Fluorescence Imaging System. The dual-NIR channel FLARE imaging system has been described in detail previously [21-23]. In this study, a 670 $\mathrm{nm}$ excitation was used at a fluence rate of $4 \mathrm{~mW} / \mathrm{cm}^{2}$, with white light (400-650 nm) at 40,000 1x. Color and NIR fluorescence images were acquired simultaneously with custom software at rates up to $15 \mathrm{~Hz}$ over a $15 \mathrm{~cm}$ diameter field of view. A pseudo-colored lime green was used for NIR fluorescence in the color-NIR merged images. The imaging system was positioned at a distance of 18 inches from the surgical field.

Ex Vivo Nerve-Specific Fluorophore Screening. Sciatic nerves from female Yorkshire pigs were harvested, fixed in 2\% paraformaldehyde (PFA), and flash frozen in optimal cutting temperature (OCT) compound using liquid nitrogen. Human sural nerve tissue was provided by the Chonnam National University Medical School in a paraffin block, and swine sciatic nerve was obtained from a Yorkshire pig prior to injecting fluorophores. Nerve tissues were cut in cross section using a cryostat at $10 \mu \mathrm{m}$ thickness onto positively charged glass slides. Tissue sections were washed once with PBS for 2 min to remove residual OCT. The tissue was then fixed to the slide with $2 \%$ PFA for $15 \mathrm{~min}$, followed by washing with PBS (3 x 5 min). A formulation was previously developed for intravenous (IV) administration and was used in the current study to incubate the fluorophores with the nerve tissue [20]. All fluorophores were mixed from the $100 \mathrm{mM}$ stock solution into the IV formulation (D5W containing 5\% Cremophor EL) at $100 \mu \mathrm{M}$ and incubated with the washed nerve tissue sections at room temperature for $20 \mathrm{~min}$. Additional IV formulation not containing fluorophore was mixed and used to wash each nerve section twice (5 min per wash) following incubation with the formulated fluorophore. The nerve section was then washed an additional 2 times with PBS (5 min per each), after which coverslips were mounted using Fluoromount-G (Southern Biotech, Birmingham, AL).

NIR fluorescence microscopy for ex vivo nerve tissue slides was performed on a Nikon Eclipse TE300 microscope system as previously described [12,20,24]. The microscope was equipped with a $100 \mathrm{~W}$ mercury light source, NIR-compatible optics, and a 
NIR-compatible 4X,10x, and 20X Plan Fluor objective lens (Nikon, Melville, NY). Images were acquired on an Orca-AG (Hamamatsu, Bridgewater, NJ). Image acquisition and analysis was performed using IPLab software (Scanalytics, Fairfax, VA). A custom filter set (Chroma Technology Corporation, Brattleboro, VT) composed of a $650 / 44 \mathrm{~nm}$ excitation filter, a $675 \mathrm{~nm}$ dichroic mirror, and a 710/50 nm emission filter were used for $700 \mathrm{~nm}$ NIR imaging, while ET710/75x, T760lpxr, and ET810/90m were used for $800 \mathrm{~nm}$ NIR imaging. For $\mathrm{BMB}$, a mercury light source was passed through a $360 / 50 \mathrm{~nm}$ BP excitation filter, a $400 \mathrm{~nm}$ LP beam splitter, and a 550/50 nm emission filter.

Animal Models. Animals were housed in an AAALAC-certified facility. Animal studies were performed under the supervision of Beth Israel Deaconess Medical Center's Institutional Animal Care and Use Committee (IACUC) in accordance with approved institutional protocols (\#101-2011 for rodents and \#046-2010 for pigs). Male CD-1 mice weighing $25 \mathrm{~g}$ and Sprague-Dawley rats weighing $\approx 250 \mathrm{~g}$ (Charles River Laboratories, Wilmington, MA) were anesthetized with $100 \mathrm{mg} / \mathrm{kg}$ ketamine and $10 \mathrm{mg} / \mathrm{kg}$ xylazine intraperitoneally (Webster Veterinary, Fort Devens, MA). Female Yorkshire pigs (E.M. Parsons and Sons, Hadley, MA) averaging $37 \mathrm{~kg}$ were induced with $4.4 \mathrm{mg} / \mathrm{kg}$ intramuscular Telazol (Fort Dodge Labs, Fort Dodge, IA), intubated, and maintained with 2\% isoflurane (Baxter Healthcare Corp., Deerfield, IL). Following anesthesia, electrocardiogram, heart rate, pulse oximetry, and body temperature were monitored throughout surgery. The number of animals $(\mathrm{N})$ used for each targeting and imaging experiment was 5 .

Intraoperative Nerve-Targeting and Imaging. For intravenous (IV) injection, each fluorophore was dissolved in D5W containing 5\% Cremophor EL at a 2 $\mathrm{mM}$ concentration. Initial in vivo screening occurred in Sprague Dawley rats by injecting $1 \mu \mathrm{mol}$ of each fluorophore based on our previous study of $\mathrm{BMB}$ (Supplementary Material: Supplementary Methods) [20]. For kinetic and dose-response studies, $50-400$ nmol of Oxazine 4 was injected intravenously into CD-1 mice and measurements taken over $24 \mathrm{~h}(\mathrm{~N}=5$ per dose and time point). Control images were acquired prior to injecting NIR fluorophores. The fluorescence signal in nervous tissues and signal-to-background ratio (SBR) compared to neighboring muscle $(\mathrm{Mu})$ was obtained over the period of imaging. For the large animal study, the optimal dose found in mice (200 - 400 nmol per mouse) was used in pigs by scaling to body surface area [25,26]. $20-40$ $\mu \mathrm{mol}(0.17-0.34 \mathrm{mg} / \mathrm{kg})$ of Oxazine 4 was injected intravenously into Yorkshire pigs and the fluores- cence images of recurrent laryngeal nerve (RLN), brachial plexus (BP), and sciatic nerve (SN) were observed in real time using the FLARE imaging system up to $4 \mathrm{~h}$ post-injection ( $\mathrm{N}=5$ subjects).

Quantitation and Statistical Analysis. The fluorescence intensity (FI) and background (BG) intensity of a region of interest (ROI) over each nerve/tissue were calculated and quantified using custom FLARE software. The FI of the RLN, BP, and $\mathrm{SN}$ at the optimal dose and time point were quantified to measure tissue-specific targeting. Adjacent tissues such as trachea (Tr), thyroid (Th), tracheoesophageal groove (TEG), muscle $(\mathrm{Mu})$, and adipose tissue (Ad) were used as background to calculate the SBR of RLN, $\mathrm{BP}$, and $\mathrm{SN}$ in pigs. Statistical analysis was carried out using a one-way ANOVA followed by Tukey's multiple comparisons test. Results were presented as mean \pm S.D. and curve fitting was performed using Prism version 4.0a software (GraphPad, San Diego, CA).

Hematoxylin and Eosin (H\&E) Histology and Immunohistochemistry (IHC). RLN, BP, and SN were extracted from rats and pigs $4 \mathrm{~h}$ post-injection and placed in $2 \%$ paraformaldehyde for $30 \mathrm{~min}$ before mounting in Tissue-Tek OCT compound (Fisher Scientific, Pittsburgh, PA) and flash-freezing in liquid nitrogen. Frozen samples were cryosectioned $(10 \mu \mathrm{m}$ per slice); 1 slide was stained with H\&E and consecutive sections were used for fluorescence microscopy and IHC. For IHC, rabbit anti-myelin basic protein (MBP; \#ab53294, Abchem) and goat anti-rabbit IRDye 800CW (\#827-08365, Li-Cor) were used to visualize myelin sheath. NIR fluorescence microscopy was performed as described above for the ex vivo assay, where Oxazine 4 and MBP antibody were imaged by $700 \mathrm{~nm}$ and $800 \mathrm{~nm}$ NIR channels, respectively.

\section{Results}

Chemical and Optical Properties. As shown in Fig. 1, a series of symmetric Oxazine derivatives having cationic pendant groups were explored for nerve-specific targeting ex vivo and in vivo. BMB (ultraviolet excitation, visible emission) was used as a positive control. Oxazine 1 and Oxazine 4 have relatively high extinction coefficients $\left(>140,000 \mathrm{M}^{-1} \mathrm{~cm}^{-1}\right)$ and a moderate Stokes shift (> $17 \mathrm{~nm}$ ) in $100 \%$ serum, pH 7.4 (Table 1 and Supplementary Material: Fig. S1). The $\log \mathrm{D}$ at $\mathrm{pH} 7.4$ of $\mathrm{BMB}$, Oxazine 170, and Oxazine 750 are higher than 3.5, while Oxazine 1 and Rhodamine 800 had values less than 1 . The $\log \mathrm{D}$ at $\mathrm{pH} 7.4$ of Oxazine 4 is 3.38 with a MW of 395.84 (Table 1 and Supplementary Material: Fig. S1). 


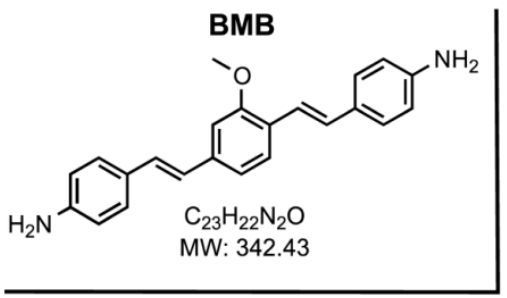

Oxazine 170

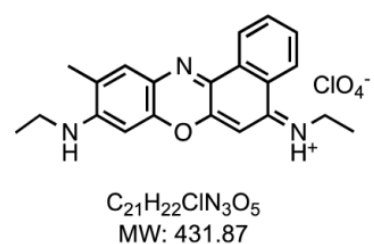

Oxazine 1 (725)<smiles>CCN(CC)c1ccc2nc3ccc(=[N+](CC)CC)cc-3oc2c1</smiles>

Oxazine 750

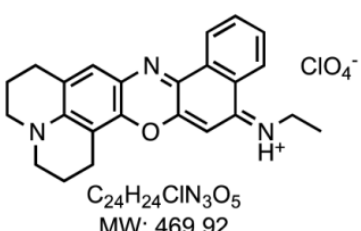

Oxazine 4<smiles>CCNc1cc2oc3cc(=[NH+]CC)c(C)cc-3nc2cc1C</smiles>

Rhodamine 800

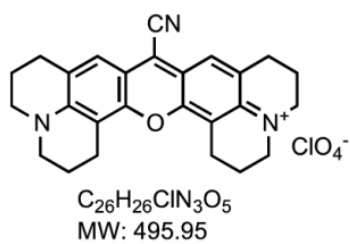

Figure I. Chemical Structure of Nerve-Targeting Fluorophores. Oxazine I is also known as Oxazine 725.

Table I. Chemical and Physical Properties of Nerve-Targeting Fluorophores.

\begin{tabular}{|c|c|c|c|c|c|c|c|c|c|c|}
\hline \multirow[t]{2}{*}{ Fluorophore } & \multirow{2}{*}{$\begin{array}{l}\text { MW } \\
\text { (Da) }\end{array}$} & \multirow{2}{*}{$\begin{array}{l}\text { LogD, } \\
\text { pH } 7.4\end{array}$} & \multirow{2}{*}{$\begin{array}{l}\text { PSA } \\
\left(\AA^{2}\right)\end{array}$} & \multirow{2}{*}{$\begin{array}{l}\text { HBD/ } \\
\text { HBA }\end{array}$} & \multirow{2}{*}{$\begin{array}{l}\text { Rotat. } \\
\text { Bonds }\end{array}$} & \multirow{2}{*}{$\begin{array}{l}\lambda \\
\left(\mathbf{M}^{-1} \mathrm{~cm}^{-1}\right)\end{array}$} & \multirow{2}{*}{$\begin{array}{l}\text { Abs } \\
(\mathrm{nm})\end{array}$} & \multirow{2}{*}{$\begin{array}{l}\text { Em } \\
(\mathrm{nm})\end{array}$} & \multicolumn{2}{|c|}{ SBR } \\
\hline & & & & & & & & & Ex vivo & In vivo \\
\hline BMB & 364.06 & 4.84 & 61.27 & $2 / 3$ & 5 & 52,800 & 397 & 498 & + & + \\
\hline Oxazine 1 & 423.89 & 0.33 & 27.84 & $0 / 3$ & 5 & 196,000 & 655 & 670 & - & - \\
\hline Oxazine 4 & 395.84 & 3.38 & 47.59 & $2 / 3$ & 3 & 143,000 & 616 & 635 & ++ & +++ \\
\hline Oxazine 170 & 431.87 & 3.94 & 47.59 & $2 / 3$ & 3 & 69,000 & 625 & 650 & + & + \\
\hline Oxazine 750 & 469.92 & 4.67 & 38.80 & $1 / 3$ & 1 & 44,000 & 676 & 684 & + & - \\
\hline Rhodamine 800 & 495.95 & 0.43 & 39.27 & $0 / 3$ & 0 & 68,000 & 697 & 710 & + & - \\
\hline
\end{tabular}

$\mathrm{MW}=$ molecular weight; PSA = polar surface area; $\mathrm{HBD} / \mathrm{HBA}=\mathrm{H}$ bond donors/acceptors; Rotat. Bonds = rotational bonds; $\lambda=$ extinction coefficient; Abs = peak absorbance; $\mathrm{Em}=$ peak emission. The SBR of each nerve relative to the neighboring adipose tissue was quantified and labeled as,$-<1 ;+, 1$ to $2 ;++, 2$ to $3 ;$ and,$+++>3$.

Ex Vivo Nerve-Specific Fluorophores Screening. $\mathrm{BMB}$, Oxazine 1, Oxazine 4, Oxazine 170, Oxazine 750, and Rhodamine 800 fluorophores were screened for nerve-specific fluorescence and undesired adipose uptake using cross sections of pig sciatic nerve (Supplementary Material: Fig. S2) and human sural nerve tissue (Fig. 2a). Fluorescence images of Oxazine derivatives were compared with the previously identified nerve-specific fluorophore BMB (positive control). Oxazine 4, Oxazine 750, and Rhodamine 800 fluorophores exhibited strong fluorescence signal throughout the cross-sectioned nerve fascicle, while Oxazine 1 and 170 showed weak nerve-specific fluorescence signal. Although the positive control BMB represented relatively high fluorescence in the nerve, the signal in epineurium and adipose tissues was even higher than the target signal (SBR $<1.0)$. From this $e x$ vivo nerve-specific screening Oxazine 4, Oxazine 750, and Rhodamine 800 were identified as the potential candidates for high performance in vivo.

In Vivo Nerve-Specific Fluorophores Screening. Initial screening of fluorophore performance in vivo utilized a single intravenous injection of $\mathrm{BMB}$ and Oxazine derivatives at a dose of $1 \mu \mathrm{mol}$ in $250 \mathrm{~g}$ SD rats $4 \mathrm{~h}$ prior to imaging (Fig. $\mathbf{2 b}$ ). To compare the difference between ex vivo and in vivo results, all fluorophores used in ex vivo screening were examined in vivo in the rat brachial plexus and sciatic nerve assays. Oxazine 4 showed the highest nerve-specific fluorescence signal and the lowest background (muscle and adipose tissue) fluorescence signal compared to other molecules and BMB. Although Oxazine 1 showed no nerve fluorescence by the ex vivo nerve screening assay, it did have a weak signal in the vicinity of nerve, but could also have been caused by high adipose tissue uptake. Based on these data, Oxazine 4 was chosen for further study.

Kinetics of Oxazine 4 Nerve Targeting in CD-1 Mice. To determine the time of peak SBR, we injected $100 \mathrm{nmol}(1.6 \mathrm{mg} / \mathrm{kg})$ of Oxazine 4 into mice and sacrificed $\mathrm{N}=5$ per time point. The fluorescence signal in the $\mathrm{BP}$ and $\mathrm{SN}$ was bright enough by $1 \mathrm{~h}$ post-injection, however, the SBR was low because of high background signal from the salivary gland, adjacent muscle, adipose tissue, and skin. The highest $\mathrm{SBR} \approx 3.0$ was achieved at $4 \mathrm{~h}$ post-intravenous injection, at which point surrounding background signal had decreased significantly $\left({ }^{*} P<0.05\right)$. Oxazine 4 uptake was higher in the brachial plexus compared to sciatic nerve, of which signal retained up to $12 \mathrm{~h}$. The background signal completely diminished after $24 \mathrm{~h}$ of a single IV injection, but the fluorescence signal in nervous tissues also decayed over time (Fig. 3a). 


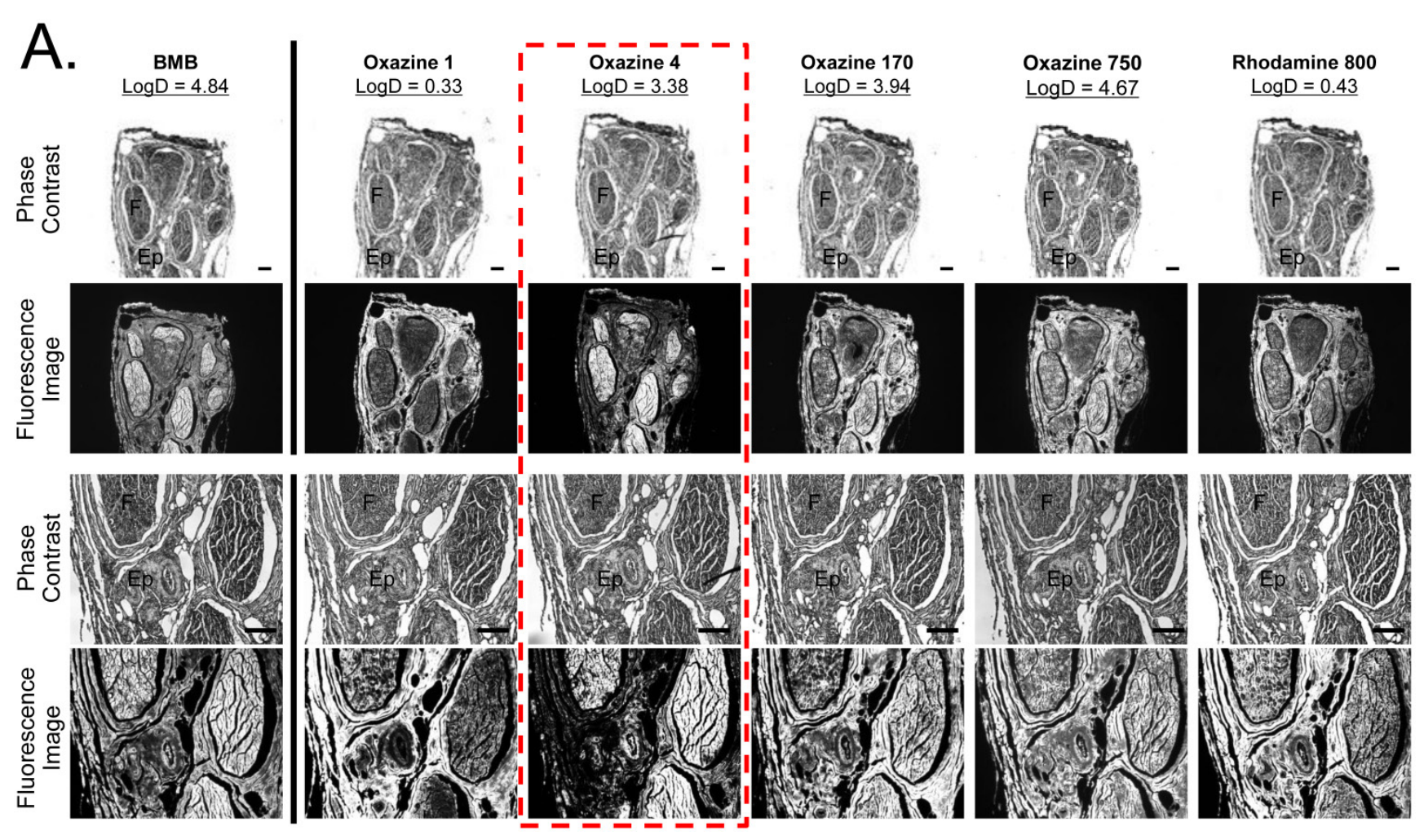

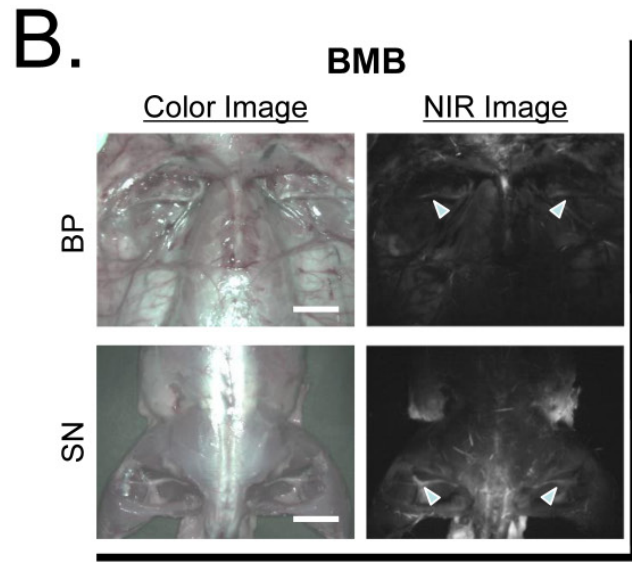

Oxazine 170
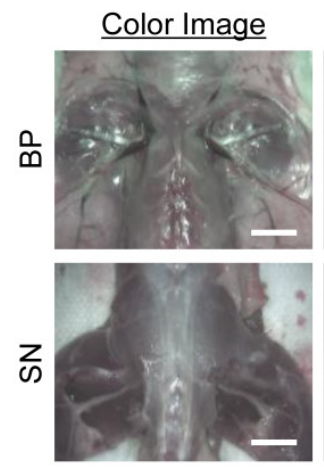

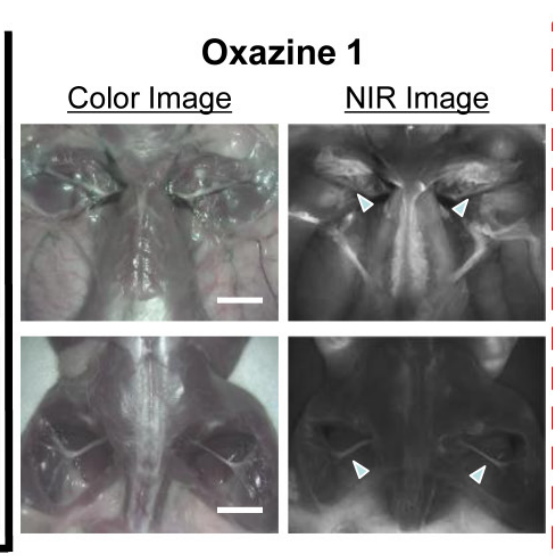

Oxazine 750
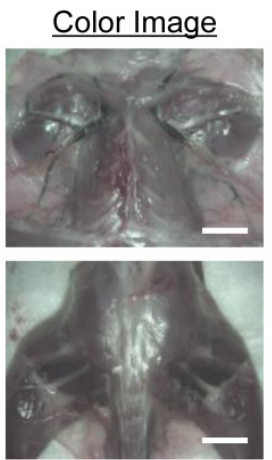

NIR Image
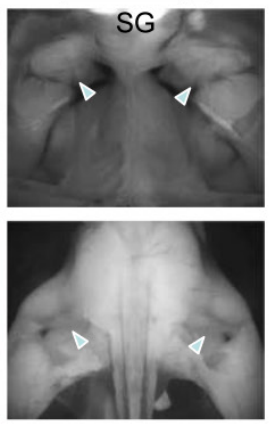

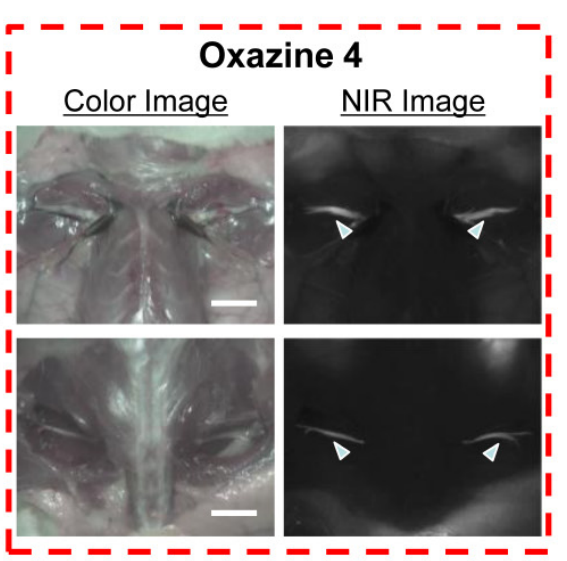

Rhodamine $\mathbf{8 0 0}$

Color Image NIR Image
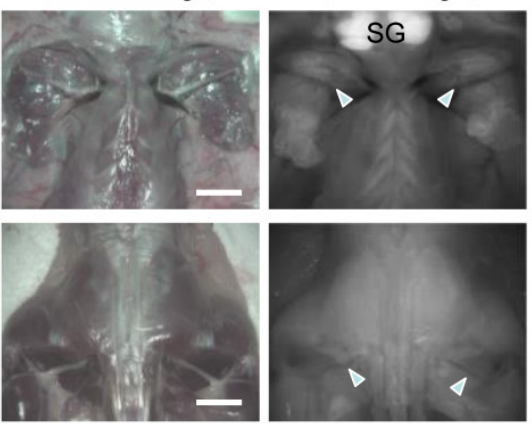

Figure 2. Ex Vivo and In Vivo Screening Assay of Fluorophores for Nerve-Specificity. A) Nerve-specific fluorescence intensity was determined using staining of human sural nerve cut in cross section and incubated with $100 \mu \mathrm{M}$ of each fluorophore. Ep = epineurium; $\mathrm{F}=$ nerve fascicle. Scale bars $=200 \mu \mathrm{m}$. The partition coefficient (logD at $\mathrm{pH} 7.4)$ was calculated using JChem calculator plugins. B) In vivo nerve-specific fluorescence in the rat brachial plexus (BP, top rows) and sciatic nerve (SN, bottom rows) $4 \mathrm{~h}$ post-injection. Arrowheads indicate fluorescence signals in nerve tissue. SG $=$ salivary gland. Scale bars $=1 \mathrm{~cm}$. All NIR fluorescence images have identical exposure and normalizations. 

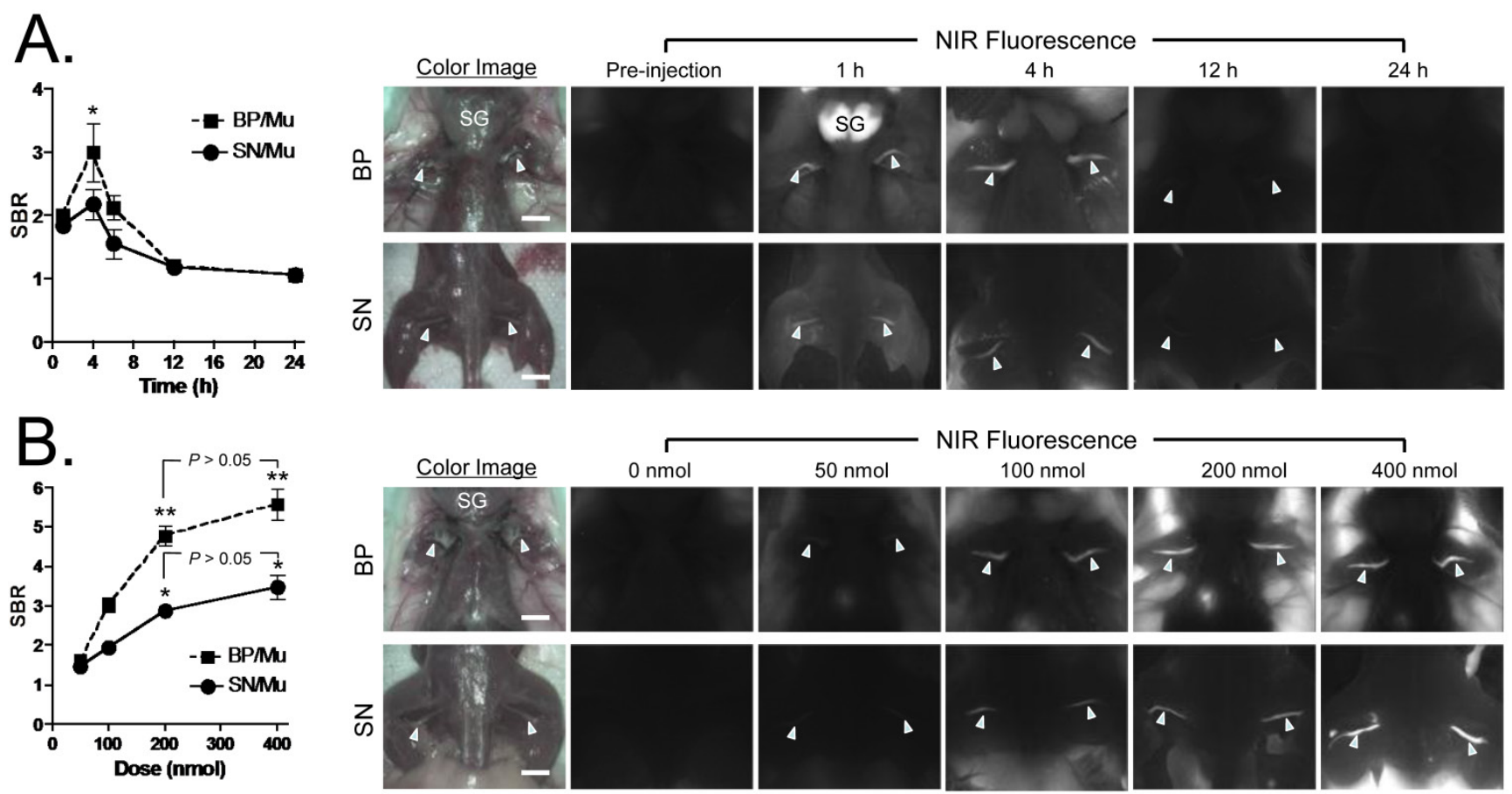

NIR Fluorescence

$50 \mathrm{nmol}$
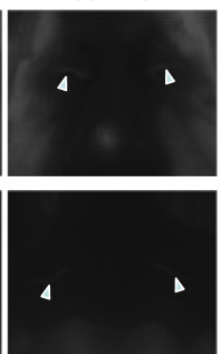
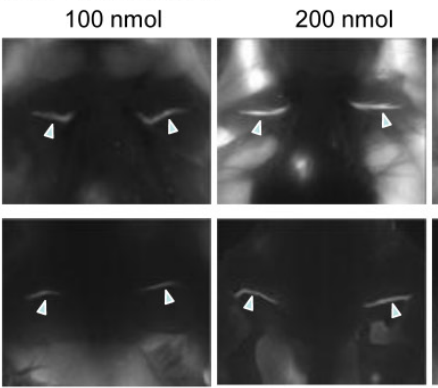

$400 \mathrm{nmol}$

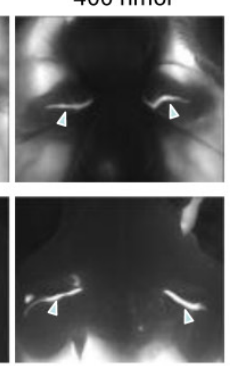

Figure 3. Kinetics and Dose-Response of Oxazine 4 Uptake in Mice A) Quantitative time-course assessment of SBR (mean \pm SEM) for brachial plexus (BP) and sciatic nerves $(\mathrm{SN}) .100 \mathrm{nmol}(1.6 \mathrm{mg} / \mathrm{kg})$ of Oxazine was injected intravenously into CD-I mice, and SBR (BP/Mu) and SBR (SN/Mu) were measured at different time points (T = 0, I, 4, 6, I2, and $24 \mathrm{~h}$ ). Arrowheads indicate fluorescence signals in nerve bundles. SG = salivary glands; Mu = muscle. B) Dose-response curve of SBR (mean \pm SEM) for BP and SN. 0, 50,100 , 200 , and $400 \mathrm{nmol}$ of Oxazine 4 were injected intravenously into CD-I mice, and SBR (BP/Mu) and SBR (SN/Mu) were measured at $4 \mathrm{~h}$ post-injection. Arrowheads indicate fluorescence signals in nerve bundles. Images are representative of $N=5$ independent experiments. $* * P<0.0 \mathrm{I}$ and $* P<0.05$. SG $=$ salivary glands; Mu $=$ muscle. Scale bars $=1$ $\mathrm{cm}$. All NIR fluorescence images have identical exposure times $(500 \mathrm{msec})$ and normalizations.

Dose-Dependence of Oxazine 4 Nerve Targeting in CD-1 Mice. Dose-dependent optimization was investigated at $4 \mathrm{~h}$ post-injection. As shown in Fig. $3 \mathbf{b}$, the fluorescence signals in the BP and SN were proportional to the injected dose in the range of $50-400$ nmol per mouse $(0.8-6.3 \mathrm{mg} / \mathrm{kg})$. The minimal dose used to highlight peripheral nerves was $50 \mathrm{nmol}$, however, a significant increase in nerve signals was found when injected with $200 \mathrm{nmol}$ of Oxazine $4\left({ }^{* *} P\right.$ $<0.01$ for $\mathrm{BP}$ and ${ }^{*} P<0.05$ for $\left.\mathrm{SN}\right)$. Although the maximum nerve signal was obtained with the IV injection of $400 \mathrm{nmol}$ Oxazine 4, the background signals in the surrounding muscle and adipose tissues were significantly increased as well. Therefore, no significant differences were observed in the SBR of BP and SN between the dose of $200 \mathrm{nmol}$ and $400 \mathrm{nmol}(P>$ 0.05).

Optimal Nerve Targeting Using Oxazine 4 in SD Rats. The BP and SN were clearly identified and highlighted after a single intravenous injection of Oxazine 4 in CD-1 mice. However, branched peripheral nerves are not identified because of difficulty in visual identification. To find out branched nerves in small animals, we injected $1 \mu \mathrm{mol}$ of Oxazine 4 intravenously into SD rats $4 \mathrm{~h}$ prior to imaging based on the optimized condition in Fig. 3. As indicated by arrowheads, small branches of main nerves including $\mathrm{BP}$ and SN could be identified in the fluorescence images that could not be distinguished visually using color video (Fig. 4a). In addition, the nervous structure was confirmed by IHC using an anti-myelin basic protein (MBP) antibody. MBP is a major component of multilayered myelin sheath and interact with the lipids in the myelin membrane [14,27]. As shown in Fig. $\mathbf{4 b}$, Oxazine 4 colocalizes with the myelinated sheath within the fascicle, which was stained by the MBP antibody.

Oxazine 4 Nerve Targeting in Large Animals. Various sites of important peripheral nerves were surgically exposed and imaged with Channel $1(\approx 700$ $\mathrm{nm}$ ) of the FLARE imaging system at 5, 15, 30, 60, 120, 180, and $240 \mathrm{~min}$ post-injection (Fig. 5). In the pre-injection images, RLN, BP, and SN show almost no autofluorescence, however, thyroid (Th) shows relatively high autofluorescence. Since the RLN is usually located in the tracheoesophageal groove (TEG), the SBR between nerves and adjacent tissues such as trachea, TEG, and Th is the key determinant for preserving the RLN during surgery. The fluorescence signal in the RLN was relatively high by $30 \mathrm{~min}$ post-injection, however, excessive signals remained in the surrounding tissues. Therefore, the maximal SBR was achieved $3 \mathrm{~h}$ post-injection (Fig. 5a). This time span should be enough to identify thin and buried RLNs and avoid nerve damage during thyroidectomy in real time. On the other hand, BP and SN were rela- 
tively easily identified at early time points, and the maximal SBR for BP and SN was achieved at $1 \mathrm{~h}$ and 2 $\mathrm{h}$ post-injection, respectively. As shown in Fig. $\mathbf{5 b}$, the signals in nervous tissue retained up to $4 \mathrm{~h}$ post-injection. In order to see the reliability of intraoperative RLN mapping for thyroid patients who get reoperation after thyroidectomy, we removed thyroid and observed RLN and surrounding tissues (Fig. 5b). The absence of thyroid improved the identification of RLN in real time, and the SBR of RLN with neighboring tissue was higher than 1.0. Additionally, nerves were resected and cryosectioned for $H \& E$ and fluorescence microscopy. As shown in Fig. 5c, NIR fluorescence microscopic images confirm bright fluorescence signal throughout the nerve, which indicates the entire endoneurium surrounding individual axon and myelinated fibers within the fascicle could be stained by the single intravenous injection of Oxazine 4.
Quantitative Structure-Activity Relationships (QSAR). Based on the QSAR screening and chemical group fingerprinting of BMB [13], we compared the 5 positive fingerprints with our new nerve-targeting fluorophores. Among the series, Oxazine 4 met the basic requirement of a balanced para configuration of the core (Supplementary Material: Fig. S4; [13]). In addition, Oxazine 4 showed the highest similarity with the 5 highest ranked fingerprints except for BMB. As shown in Fig. 6, when compared to the chemical structure of BMB and its constituent groups, Oxazine 4 also ranked top in most cases. Rhodamine 800 scored higher for some fragments, but its uptake in nerve was low because of its low $\log \mathrm{D}$ at $\mathrm{pH} 7.4(0.43)$. Conversely, Oxazine 170 and Oxazine 750, which have high $\log \mathrm{D}$ at $\mathrm{pH} 7.4$ values (3.94 and 4.67, respectively) showed strong adipose uptake both ex vivo and in vivo.

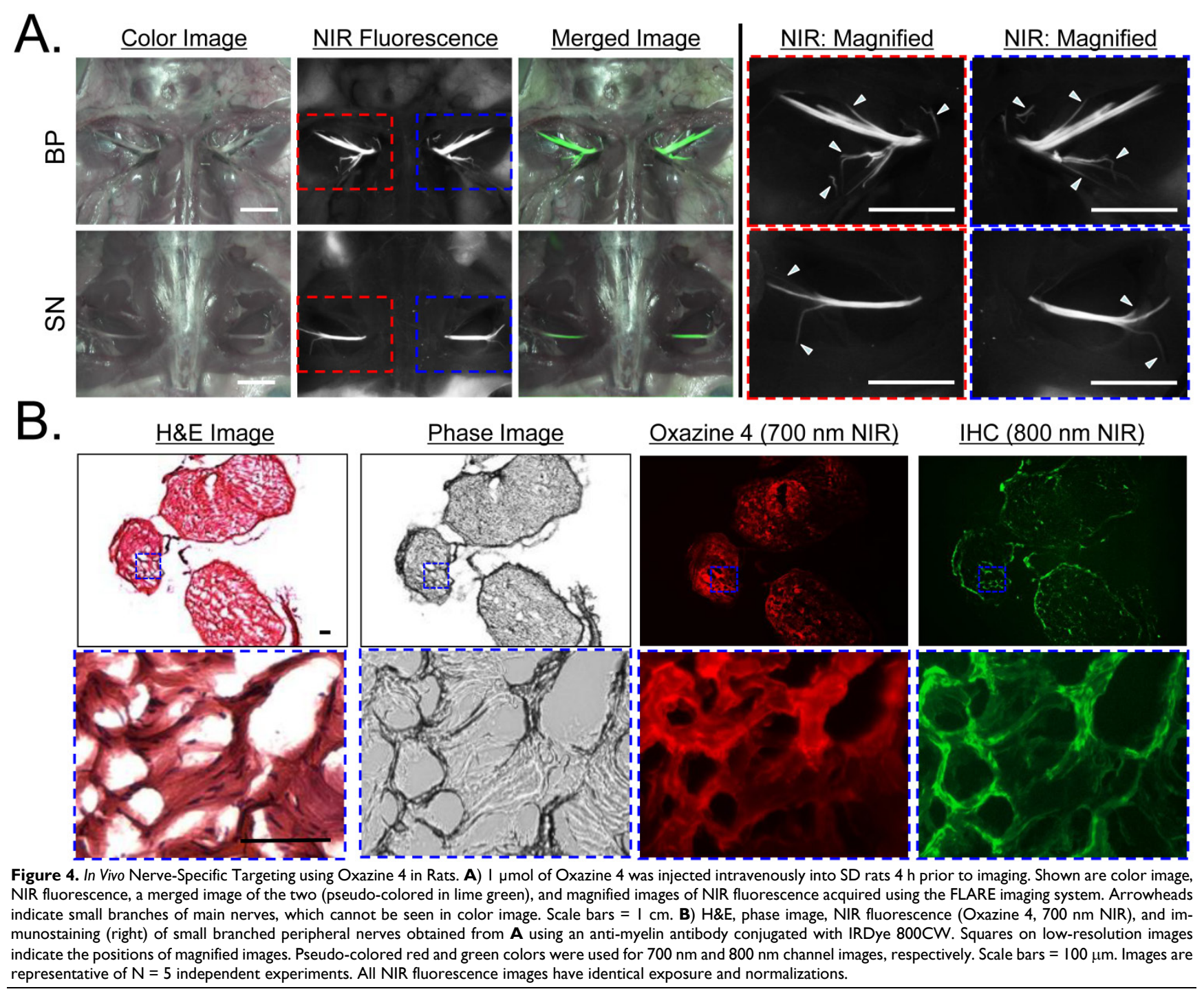



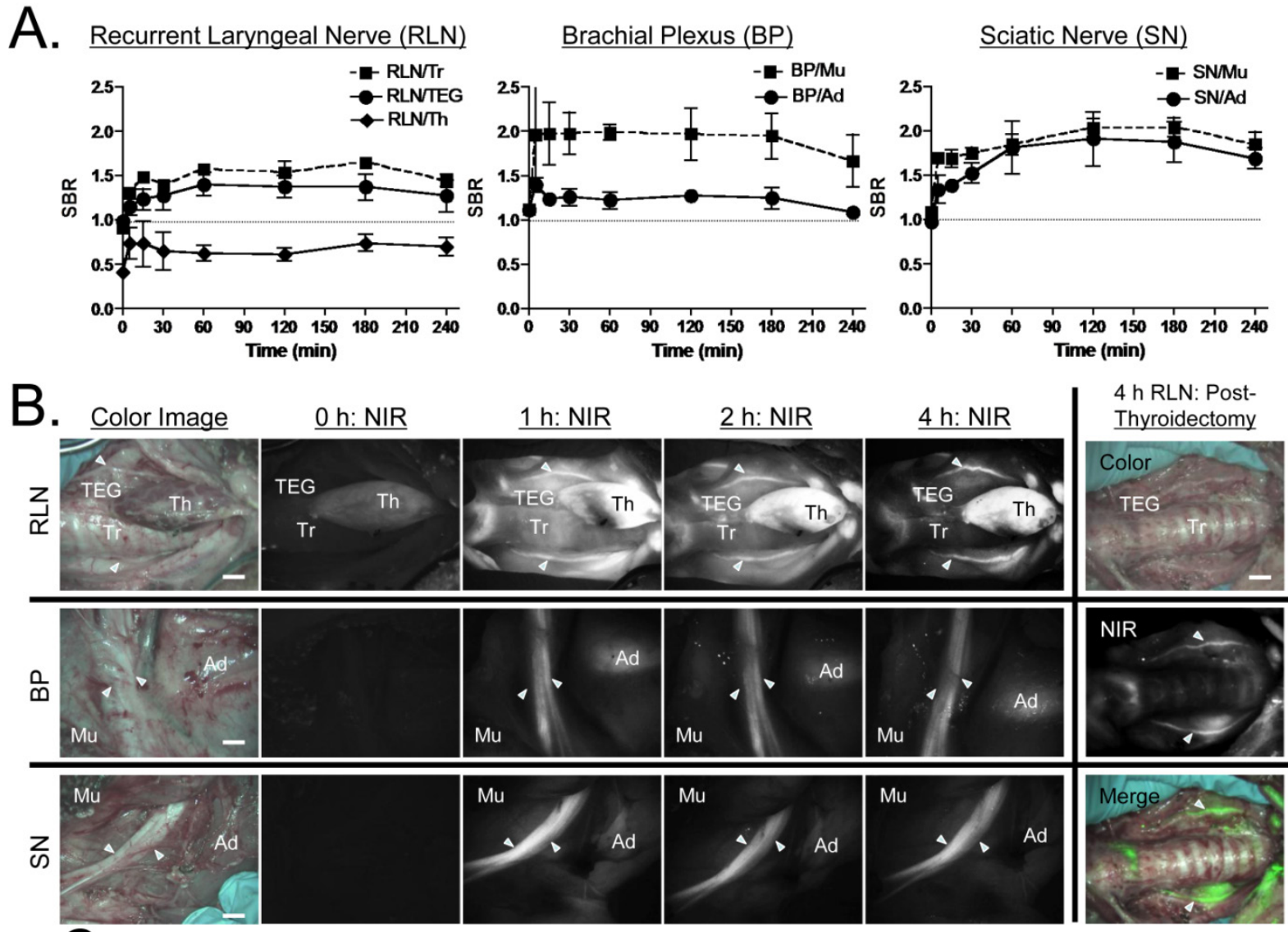

C.
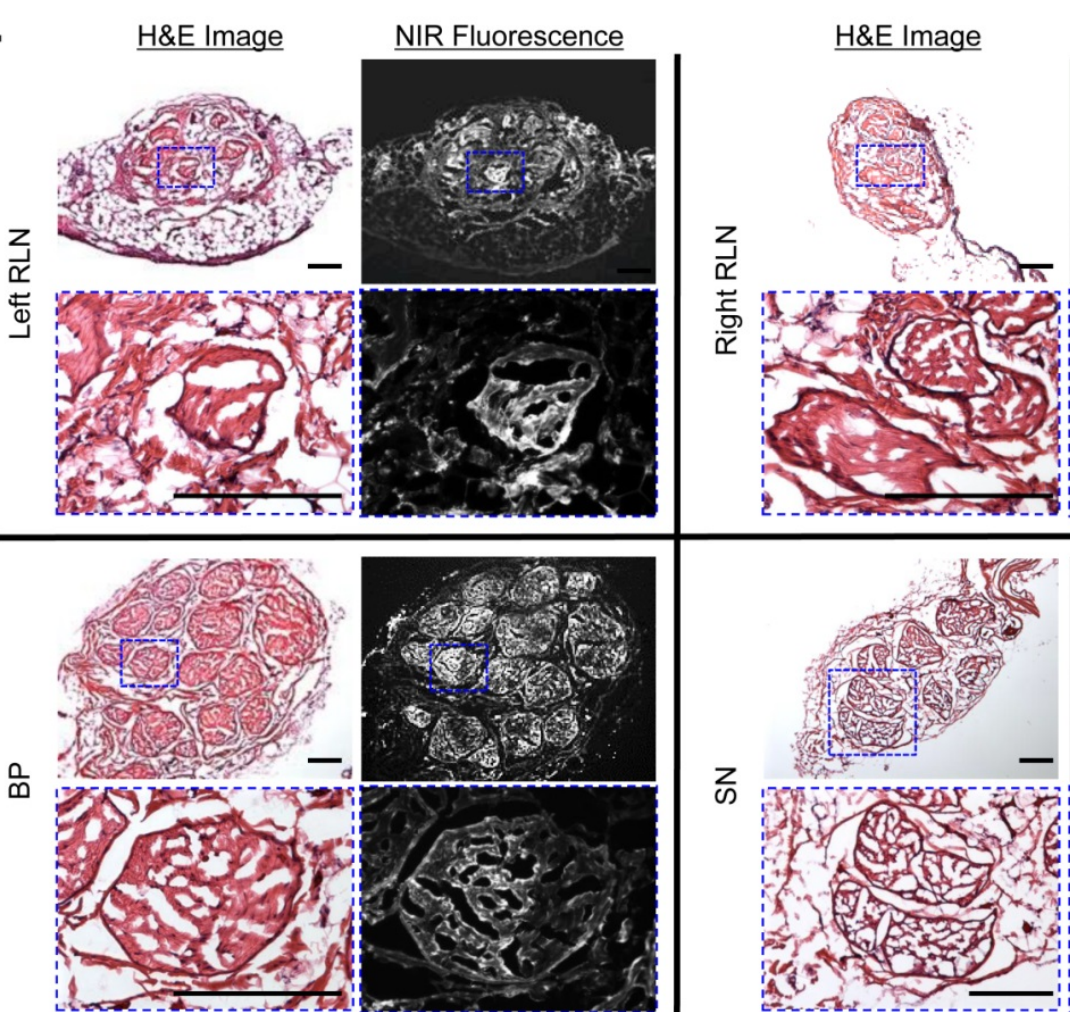

NIR Fluorescence

Figure 5. Kinetics of Oxazine 4 Uptake in Pig Nerves. A) Quantitative time course assessment of SBR (mean \pm SEM) for recurrent laryngeal nerve (RLN), brachial plexus (BP), and sciatic nerve (SN). $20 \mu \mathrm{mol}(0.25 \mathrm{mg} / \mathrm{kg})$ of Oxazine 4 was injected intravenously into $35 \mathrm{~kg}$ Yorkshire pigs, and the SBR of nerves to the specified surrounding tissue was measured at different time points $(T=0,15,30,60,120,180$, and $240 \mathrm{~min}$ ). B) Real-time intraoperative fluorescence images of RLN (top), BP (middle), and SN (bottom) acquired at different time points ( $T=0, I, 2$, and $4 \mathrm{~h}$ ). Thyroid was resected (thyroidectomy) after $4 \mathrm{~h}$, and RLN was highlighted without signal interruption. Ad = adipose tissue; Mu = muscle; $T E G=$ tracheoesophageal groove; $T h=$ thyroid; $T r=$ trachea. Scale bars $=1 \mathrm{~cm}$. C) Fluorescence microscopic images of cryosectioned RLN, BP, and SN. Samples were obtained after injecting $20 \mu \mathrm{mol}$ of Oxazine 4 into Yorkshire pigs $4 \mathrm{~h}$ prior to resection. Shown are representative images $(\mathrm{N}=5)$ of H\&E (left) and NIR fluorescence (right) images. Blue squares on H\&E images indicate the positions of magnified images. Scale bars $=100 \mu \mathrm{m}$. All NIR fluorescence images have identical exposure times and normalizations. 

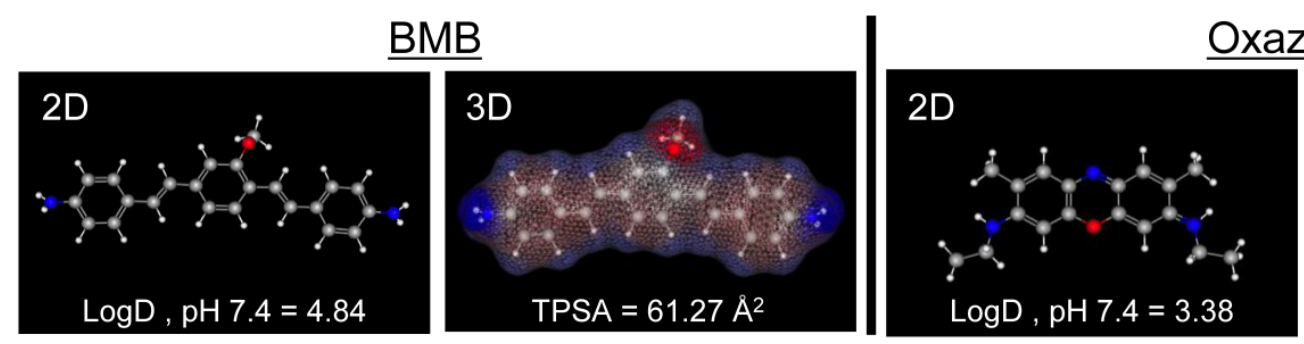

\section{Oxazine 4}
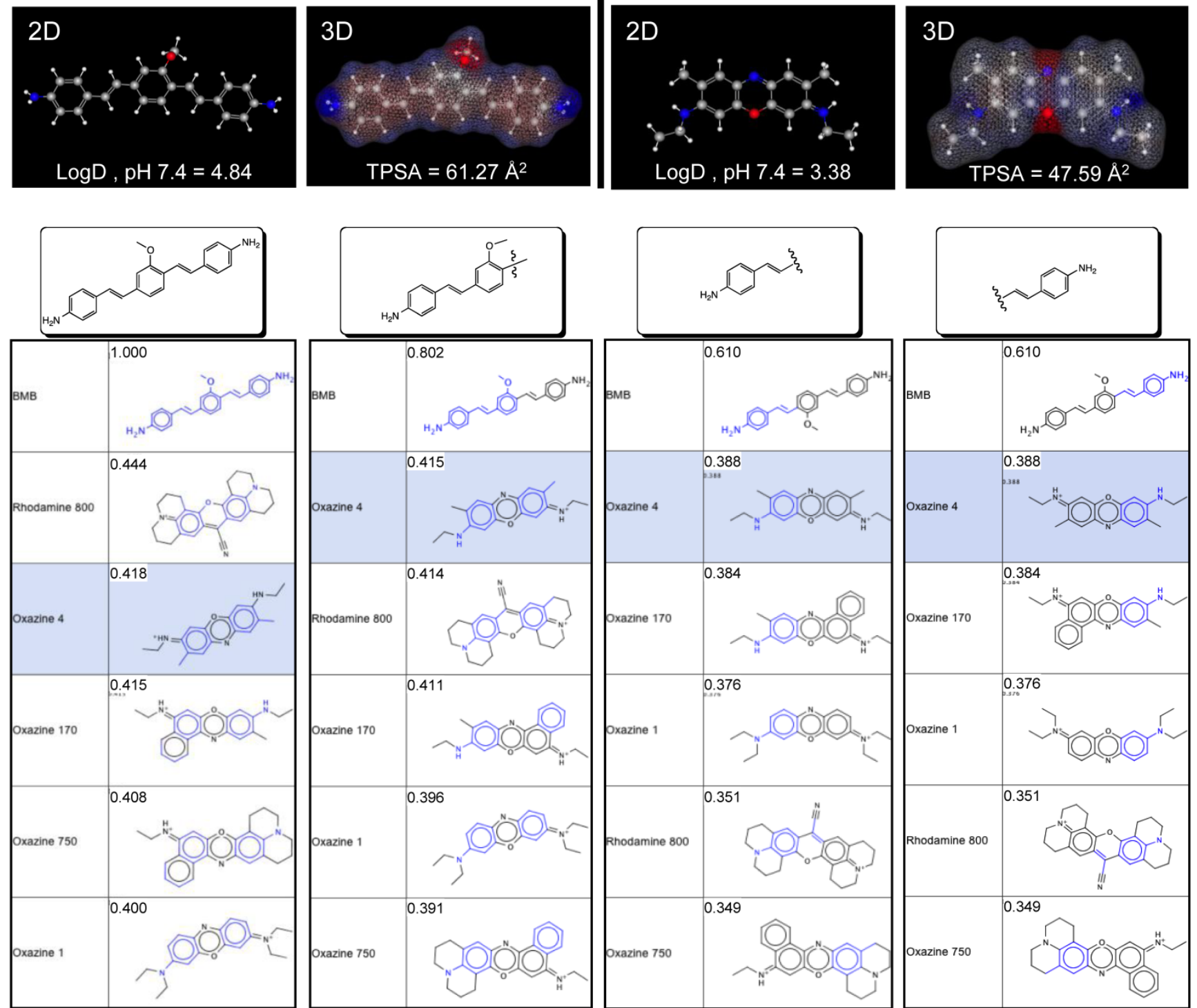

Figure 6. Chemical Similarities between Non-NIR and NIR Nerve-Specific Contrast Agents. BMB (non-NIR) and Oxazine 4 (NIR) are shown as 2D energy-minimized structures, 3D structures with charge (red = negative; blue $=$ positive) and hydrophobicity (gray) distributions, and in comparison to BMB and its sub-structures. Similarity was calculated using the chemical hashed fingerprint method with a Normalized Euclidean screening configuration (Instant JChem v 6.04, ChemAxon).

\section{Discussion}

Image-guided surgery is now in routine clinical use to improve surgical fields by using interventional MRI, ultrasound, and electrophysiological recordings. However, limitations remain in regard to visualizing and guiding thin or buried nerves due to the lack of nerve-specific imaging [28]. Most recently, Tsien and colleagues made a significant improvement in nerve-specific targeting and imaging, where an engineered peptide FAM-NP41 was used to label in vivo animal nerves and resected human RLN with relatively high affinity [16]. Since small molecules are about 10-1000 fold smaller than peptides and proteins, however, they will need at least 10-fold less dosage if the affinity is identical. It should be noted that the optimal dose of the molecules we describe in this study is 10-50 fold lower than previously described nerve-specific fluorophores [11-13,16] and corresponds to a human equivalent dose of only 0.25 $\mathrm{mg} / \mathrm{kg}[25,26]$, which is consistent with the clinically-approved dose of the NIR fluorophore indocyanine green (ICG). Importantly, at this dose range, Oxazine 4 does not stain the central nervous system, and did not show apparent impairment of motor function and sensory integrity during the $24 \mathrm{~h}$ post-injection (data not shown).

Furthermore, the modification of physicochemical properties of small molecules is relatively easy for real-time image guided surgery [29]. To develop an ideal nerve-specific contrast agent for image-guided surgery, we need to consider the following criteria: 1) 
$\log \mathrm{D}$ at $\mathrm{pH} 7.4$ between 0.5 to 3 and molecular weight $<500 \mathrm{Da}$ to maximize blood-nerve-barrier (BNB) penetration, 2) excitation and emission wavelengths in the NIR window, and 3) prolonged retention in the nerve tissue [30]. To the best of our knowledge, Oxazine 4 is the first small molecule described to date that closely meets all criteria. Based on its chemical similarity to $\mathrm{BMB}$, Oxazine 4 likely targets myelin or a closely associated molecule. Nonetheless, it should be considered a prototype molecule because the optical property of Oxazine 4 is not in the true NIR window. Additionally, biodistribution and clearance should be considered because they mediate the fate of contrast agents in vivo such as how long after intravenous injection a contrast agent reaches peak SBR, and how long the signal lasts. The nerve-specific signals may vary depending on the species because of different biodistribution, metabolism, and pharmacokinetics. However, the pharmacokinetic data presented in both small and large animals suggest that nerve penetration is rapid, blood and tissue clearance is rapid, yet retention in nerves is strong enough to permit an SBR $>1$ for 3-4 hours, which is good enough for complicated nerve surgery. After optimizing the optical and chemical properties described above, attention will need to be turned to nerve affinity. Increasing affinity will further improve nerve retention while also increasing signal strength and the imaging period.

In summary, near-infrared fluorescent, nerve-specific small molecules could be considered the Holy Grail in the field of image-guided surgery. Although our work does not conclude the quest, it lays the foundation for another push towards the goal by helping surgeons to avoid nerve damage on a patient-specific basis.

\section{Supplementary Material}

Supplementary methods, Fig.S1 - Fig.S5.

http://www.thno.org/v04p0823s1.pdf

\section{Acknowledgments}

We thank Rita G. Laurence for assistance with animal surgery, David Burrington, Jr. for editing, and Eugenia Trabucchi for administrative assistance. This study was supported by the following grants from the National Institutes of Health: NCI BRP grant \#R01-CA-115296 (JVF), NIBIB grant \#R01-EB-010022 (JVF and HSC), and NIBIB grant \#R01-EB-011523 (HSC and JVF), and a grant from the Dana Foundation in Brain and Immuno-Imaging (HSC). The content expressed is solely the responsibility of the authors and do not necessarily represent the official views of the National Institutes of Health.

\section{Conflict of interest}

FLARE $^{\text {TM }}$ technology is owned by Beth Israel Deaconess Medical Center, a teaching hospital of Harvard Medical School. Dr. Frangioni has three for-profit companies, Curadel, Curadel ResVet Imaging, and Curadel Surgical Innovations, which have optioned FLARE ${ }^{\mathrm{TM}}$ technology from Beth Israel Deaconess Medical Center and may someday be licensees.

\section{References}

1. Cohen AJ, Katz MG, Katz R, Mayerfeld D, Hauptman E, Schachner A. Phrenic nerve injury after coronary artery grafting: is it always benign? Ann Thorac Surg. 1997; 64: 148-53.

2. Chaudhary IA, et al. Recurrent laryngeal nerve injury: an experience with 310 thyroidectomies. J Ayub Med Coll Abbottabad. 2007; 19: 46-50.

3. Clough RW, Neese SL, Sherill LK, Tan AA, Duke A, Roosevelt RW, et al. Cortical edema in moderate fluid percussion brain injury is attenuated by vagus nerve stimulation. Neuroscience. 2007; 147: 286-93.

4. Shi C, Flanagan SR, Samadani U. Vagus nerve stimulation to augment recovery from severe traumatic brain injury impeding consciousness: a prospective pilot clinical trial. Neurol Res. 2013; 35: 263-76.

5. Lang BH, Lo CY, Chan WF, Lam KY, Wan KY. Staging systems for papillary thyroid carcinoma: a review and comparison. Ann Surg. 2007; 245: 366-78.

6. Snyder SK, Lairmore TC, Hendricks JC, Roberts JW. Elucidating mechanisms of recurrent laryngeal nerve injury during thyroidectomy and parathyroidectomy. J Am Coll Surg. 2008; 206: 123-30.

7. Myssiorek D. Recurrent laryngeal nerve paralysis: anatomy and etiology. Otolaryngol Clin North Am. 2004; 37: 25-44.

8. Wu C, Wei J, Tian D, Feng Y, Miller RH, Wang Y. Molecular probes for imaging myelinated white matter in CNS. J Med Chem. 2008; 51: 6682-8.

9. Wu C, Tian D, Feng Y, Polak P, Wei J, Sharp A, et al. A novel fluorescent probe that is brain permeable and selectively binds to myelin. J Histochem Cytochem. 2006; 54: 997-1004.

10. Stankoff B, Wang Y, Bottlaender M, Aigrot MS, Dolle F, Wu C, et al. Imaging of CNS myelin by positron-emission tomography. Proc Natl Acad Sci U S A. 2006; 103: 9304-9.

11. Meyers JR, MacDonald RB, Duggan A, Lenzi D, Standaert DG, Corwin JT, et al. Lighting up the senses: FM1-43 loading of sensory cells through nonselective ion channels. J Neurosci. 2003; 23: 4054-65.

12. Gibbs-Strauss SL, Vooght C, Fish KM, Nasr KA, Siclovan TM, Barnhardt NE, et al. Molecular imaging agents specific for the annulus fibrosus of the intervertebral disk. Mol Imaging. 2010; 9: 128-40.

13. Gibbs SL, Xie Y, Goodwill HL, Nasr KA, Ashitate Y, Madigan VJ, et al. Structure-activity relationship of nerve-highlighting fluorophores. PLoS One. 2013; 8: e73493.

14. Cotero VE, Siclovan T, Zhang R, Carter RL, Bajaj A, LaPlante NE, et al. Intraoperative fluorescence imaging of peripheral and central nerves through a myelin-selective contrast agent. Mol Imaging Biol. 2012; 14: 708-17.

15. Nakayama A, Bianco AC, Zhang CY, Lowell BB, Frangioni JV. Quantitation of brown adipose tissue perfusion in transgenic mice using near-infrared fluorescence imaging. Mol Imaging. 2003; 2: 37-49.

16. Whitney MA, Crisp JL, Nguyen LT, Friedman B, Gross LA, Steinbach P, et al. Fluorescent peptides highlight peripheral nerves during surgery in mice. Nat Biotechnol. 2011; 29: 352-6.

17. Wu AP, Whitney MA, Crisp JL, Friedman B, Tsien RY, Nguyen QT. Improved facial nerve identification with novel fluorescently labeled probe. The Laryngoscope. 2011; 121: 805-10.

18. Gustafson TP, Yan Y, Newton P, Hunter DA, Achilefu S, Akers WJ, et al. A NIR dye for development of peripheral nerve targeted probes. Med Chem Commun. 2012; 3: 685-90.

19. Vahrmeijer AL, Hutteman M, van der Vorst JR, van de Velde CJ, Frangioni JV. Image-guided cancer surgery using near-infrared fluorescence. Nat Rev Clin Oncol. 2013; 10: 507-18

20. Gibbs-Strauss SL, Nasr KA, Fish KM, Khullar O, Ashitate Y, Siclovan TM, et al. Nerve-highlighting fluorescent contrast agents for image-guided surgery. Mol Imaging. 2011; 10: 91-101.

21. Troyan SL, Kianzad V, Gibbs-Strauss SL, Gioux S, Matsui A, Oketokoun R, et al. The FLARE intraoperative near-infrared fluorescence imaging system: a first-in-human clinical trial in breast cancer sentinel lymph node mapping. Ann Surg Oncol. 2009; 16: 2943-52.

22. Gioux S, Kianzad V, Ciocan R, Gupta S, Oketokoun R, Frangioni JV. High-power, computer-controlled, light-emitting diode-based light sources for fluorescence imaging and image-guided surgery. Mol Imaging. 2009; 8: 156-65.

23. Ashitate Y, Stockdale A, Choi HS, Laurence RG, Frangioni JV. Real-time simultaneous near-infrared fluorescence imaging of bile duct and arterial anatomy. J Surg Res. 2012; 176: 7-13 
24. Ashitate Y, Kim SH, Tanaka E, Henary M, Choi HS, Frangioni JV, et al. Two-wavelength near-infrared fluorescence for the quantitation of drug antiplatelet effects in large animal model systems. J Vasc Surg. 2012; 56: 171-80.

25. Kelley KW, Curtis SE, Marzan GT, Karara HM, Anderson CR. Body surface area of female swine. J Anim Sci. 1973; 36: 927-30.

26. Reagan-Shaw S, Nihal M, Ahmad N. Dose translation from animal to human studies revisited. Faseb J. 2008; 22: 659-61.

27. Deber CM, Reynolds SJ. Central nervous system myelin: structure, function, and pathology. Clin Biochem. 1991; 24: 113-34.

28. Peters TM. Image-guidance for surgical procedures. Phys Med Biol. 2006; 51: R505-40.

29. Cotero VE, Siclovan T, Zhang R, Carter RL, Bajaj A, LaPlante NE, et al. Intraoperative fluorescence imaging of peripheral and central nerves through a myelin-selective contrast agent. Mol Imaging Biol. 2012; 14: 708-17.

30. Pajouhesh H, Lenz GR. Medicinal chemical properties of successful central nervous system drugs. NeuroRx. 2005; 2: 541-53. 